\title{
Digestibilité de la paille traitée à la soude chez le poney
}

\author{
R. WOLTER, J.P. VALETTE et G. MOREL-GARAY \\ avec la collaboration technique de M. Tournoux \\ Laboratoire de Nutrition et Alimentation \\ Ecole nationale vétérinaire d'Alfort \\ F 94704 Maisons-Alfort Cedex
}

\section{Résumé}

L'étude, chez 4 poneys, de la digestibilité de la paille broyée, agglomérée et traitée à la soude, distribuée à volonté $\left(40\right.$ à $94 \mathrm{~g} / \mathrm{kg} \mathrm{PV}^{0,75}$ ) ou en quantité restreinte (près de $\left.42 \mathrm{~g} / \mathrm{kg} \mathrm{PV} \mathrm{PV}^{0,75}\right)$, seule ou avec un complément de tourteau de soja (14 p. $100 \mathrm{du}$ régime, pour que celui-ci atteigne 10,6 p. $100 \mathrm{MAT}$ ), montre que :

- le traitement à la soude augmente nettement la digestibilité de la paille (CUD de la matière organique : 47,5 p. 100 , au lieu de 28,8 p. 100 avec la paille native);

- la restriction de la consommation de paille sodée en modifie très peu la digestibilité chez le poney;

- la complémentation en tourteau de soja,

- avec la paille sodée en libre consommation, n'améliore pratiquement pas la digestibilité de la ration totale, et tend donc à abaisser la digestibilité propre de la paille,

- avec la paille sodée distribuée en quantité limitée, élève significativement la digestibilité du régime global, sans réhausser sensiblement la digestibilté de la paille elle-même.

\section{Introduction}

La paille a une forte teneur en cellulose brute $(\simeq 40$ p. 100) et en lignine (10 p. 100); elle contient peu de matières azotées $(2-4$ p. 100). La cellulose, en principe digestible, est liée, avec les hémicelluloses, à la lignine non digestible. De ce fait, la digestibilité de la cellulose brute de la paille n'atteint que 35 à 55 p. 100 chez les ruminants (JACKsON, 1977).

Une augmentation de cette digestibilité peut être attendue d'une amélioration de l'équilibre alimentaire, par adjonction d'azote et de minéraux qui activent la cellulolyse microbienne, au moins chez les ruminants et dans des conditions d'apports limités en paille, et du maintien d'un $\mathrm{pH}$ ruminal assez voisin de la neutralité. Elle résulte aussi de la fragmentation physique qui accroît la surface d'attaque microbienne. Elle est encore davantage renforcée par les traitements alcalins. Ceux-ci 
désorganisent la lignine et la solubilisent très partiellement; surtout ils attaquent les hémicelluloses dont la solubilisation peut atteindre 70 p. 100 ; ils épargnent à peu près complètement la cellulose proprement dite qui, grandement libérée de ses liaisons avec la lignine et les hémicelluloses, est alors beaucoup plus sensible aux fermentations microbiennes (JACKSON, 1977).

Les traitements pratiques à la soude ou parfois à l'ammoniaque ont été bien étudiés quant à leurs modalités et à leurs résultats positifs chez les ruminants (Jouany, 1975 ; Copper, Morgan \& Parr, 1977 ; Jackson, 1977 ; Dulphy \& GomezCabrera, 1977 ; van Hoecke \& Cottyn, 1979; Dulphy \& ANDrieu, 1980). La productivité des animaux ne peut qu’en être renforcée; leur santé et la qualité de leurs productions ne sont nullement altérées; seule une éventuelle pollution sodique des sols par les urines est quelque peu plausible (VoIgt \& Piatkowski, 1974).

Ces traitements se justifient déjà largement dans les pays développés, lors de pénurie de bons fourrages et pour valoriser de multiples sous-produits cellulosiques (des industries du bois et du papier ou du secteur agro-alimentaire), tout en tentant de réduire alors l'emploi des concentrés. A plus forte raison, ils méritent d'être étendus dans les pays en voie de développement où les pailles et autres fourrages grossiers (bagasses, enveloppes de fruits ou de graines, sciure, tiges ligneuses...) représentent d'abondantes disponibilités (JACKSON, 1978).

Chez les équidés, par comparaison aux ruminants, les facultés de digestion de la cellulose sont nettement plus faibles (Hintz \& SCHRyver, 1972), mais comme il n'apparaît pas de besoin propre en fibres longues, les fourrages traditionnels peuvent aisément être remplacés par des sources cellulosiques très diverses telles que maïsfourrage, farine de luzerne, son de blé, pellicules de soja, marcs de pommes et de raisins, tourteau de pépins de raisins ou paille de blé (Wolter, Durix \& LetourNEAU 1,975; Wolter et al., 1979, 1980). En outre, à propos de ces derniers fourrages pauvres, ont été bien vérifiés les avantages d'une complémentation azotée (JOHNSON \& HART, 1974), ainsi que d'un traitement à la soude ou à l'ammoniaque (Slagswold, Hintz \& Schryver, 1978 ; Mundt, 1978; Meyer \& Mundt, 1980 ; Meyer, Schmidt \& Guldenhaupt, 1981).

Dans le présent essai, nous évaluons la digestibilité de la paille traitée à la soude, distribuée en quantité libre ou restreinte, et complétée ou non en tourteau de soja.

\section{Matériel et méthodes}

\section{A. Aliments}

La composition centésimale des aliments utilisés - paille traitée à la soude (P.S.) et tourteau de soja (T.S.) — ainsi que du régime - est rapportée dans le tableau 1.

De la paille de blé a été traitée avec 5 p. 100 de soude, taux qui permet la meilleure valorisation du produit (FEIST, BAKER \& TARKow, 1970). Elle est préparée par la C.O.F.N.A. sous l'appellation commerciale de NIS et se présente sous forme de granulés jaune clair de 8 à $9 \mathrm{~mm}$, peu friables. Elle a une énergie brute de $15,8 \mathrm{~kJ} / \mathrm{g}$. 


\section{TABleaú 1}

Composition des matières premières et du régime étudié.

Composition of raw materials and diet.

\begin{tabular}{|c|c|c|c|c|c|c|}
\hline \multirow{2}{*}{$\because$} & \multicolumn{3}{|c|}{ Paille - Straw } & \multicolumn{2}{|c|}{$\begin{array}{l}\text { Tourteau de soja } \\
\text { Soybean meal }\end{array}$} & \multirow{2}{*}{$\begin{array}{l}\text { Paille sodée } \\
(0,86) \\
+ \text { tourteau } \\
\text { de soja } \\
(0,14) \\
\text { NaOH straw } \\
+ \text { soybean } \\
\text { meal } \\
\text { a }\end{array}$} \\
\hline & a & $\mathrm{b}$ & c & a & $\mathrm{b}$ & \\
\hline $\begin{array}{l}\text { Matière sèche (p. 100) } \\
\text { Dry matter (p. 100) }\end{array}$ & 90.2 & 88,0 & 88.9 & 89,1 & 88,3 & 89,1 \\
\hline $\begin{array}{l}\text { En p. } 100 \text { de M.S. } \\
\text { In p. } 100 \text { of D.M. }\end{array}$ & & & & & & \\
\hline $\begin{array}{l}\text { Matières azotées totales } \\
(\mathrm{N} \times 6,25) \ldots \ldots \ldots \ldots \\
\text { Crude protein }\end{array}$ & 3,31 & 3,80 & 3,30 & 52,8 & 53,8 & 10,4 \\
\hline $\begin{array}{l}\text { Cellulose Weende } \ldots \ldots \ldots \ldots \\
\text { Crude cellulose }\end{array}$ & 39,4 & 42,0 & 39,6 & 3,4 & 4,5 & 32,8 \\
\hline Lignocellulose (A.D.F.) ... & 518 & 51,0 & 52,1 & 10,1 & - & 42,7 \\
\hline Lignine (A.D.L.) $\ldots \ldots \ldots \ldots \ldots$ & 7,9 & 7,0 & 8,1 & 2,3 & - & 7,7 \\
\hline $\begin{array}{l}\text { Cellulose «vraie» (A.D.F.-Li- } \\
\text { gnine) } \ldots \ldots \ldots \ldots \ldots \ldots \ldots \\
\text { True cellulose }\end{array}$ & 43.9 & 44,0 & 45,6 & 7,8 & - & 35,0 \\
\hline $\begin{array}{l}\text { Matière organique } \ldots \ldots \ldots \ldots \\
\text { Organic matter }\end{array}$ & 89,1 & 92,0 & 94.4 & 92,7 & 93,3 & 89,2 \\
\hline $\begin{array}{l}\text { Matières minérales - Ash } \ldots \ldots \\
\text { dont : }\end{array}$ & 10.9 & 8,0 & 5,6 & 7.3 & 6,7 & 10,8 \\
\hline Ca'cium $\ldots \ldots \ldots \ldots \ldots$ & 0,37 & 0,35 & 0,54 & 0.32 & 0,33 & 0,36 \\
\hline Phosphore - Phosphorus ...... & 0,08 & 0,10 & 0,08 & 0.70 & 0,75 & 0,17 \\
\hline Sodium & 2,87 & 0,02 & 一 & - & - & 2,49 \\
\hline
\end{tabular}

(a) Analyses effectuées au laboratoire. Analyses made at the laboratory.

(b) Réf. I.N.R.A., 1978 ; paille de blé et tourteau de soja 50. Wheat straw and soybean meal 50.

(c) Wolter et al., 1979 ; paille de blé. Wheat straw. 
Le régime comporte 86 p. 100 de paille sodée et 14 p. 100 de tourteau de soja, de façon à atteindre un taux de MAT voisin de 10 p. 100.

\section{B. Animaux}

Quatre poneys mâles adultes, croisés Shetland, dont les poids varient de 180 à $200 \mathrm{~kg}$, ont été placés dans des cages à digestibilité munies de mangeoires amovibles et d'abreuvoirs automatiques. Un dispositif destiné à la récolte des urines a été ajouté. Les animaux ont été pesés au début et à la fin de chaque période expérimentale et ont pris de l'exercice pendant les périodes d'adaptation.

Au cours des quatre périodes expérimentales suivantes, ils ont reçu les régimes, soit en quantité limitée correspondant à la consommation volontaire individuelle la plus faible $\left(\simeq 40 \mathrm{~g} / \mathrm{kg} \mathrm{PV} \mathrm{PV}^{0,75 / \mathrm{j})}\right.$ en deux repas quotidiens, soit en libre disposition continue conduisant à une consommation fréquemment renouvelée et globalement

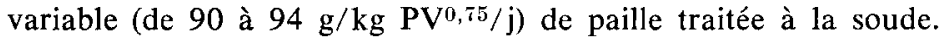

\begin{tabular}{|c|c|c|c|}
\hline $\begin{array}{l}\text { Périodes } \\
\text { Periods }\end{array}$ & $\begin{array}{l}\text { Régimes } \\
\text { Diets }\end{array}$ & $\begin{array}{l}\text { Niveaux d'alimentation } \\
\text { Level of feeding }\end{array}$ & $\begin{array}{l}\text { Adaptation } \\
\text { (en jours) } \\
\text { (in days) }\end{array}$ \\
\hline $\mathrm{I} \ldots \ldots \ldots \ldots \ldots$ & P.S. & ad libitum & 15 \\
\hline $\mathrm{II} \ldots \ldots \ldots \ldots \ldots$ & P.S. + T.S. & ad libitum & 21 \\
\hline$\ldots \ldots \ldots \ldots$ & P.S. + T.S. & limité - restricted & 8. \\
\hline IV .. & P.S. & limité - restricted & 15 \\
\hline
\end{tabular}

Le tableau 2 récapitule les poids des animaux et les quantités ingérées pendant ces périodes. Il faut peut-être mettre sur le compte de l'adaptation à la paille traitée le fait que le poney $\mathrm{C}$ ait consommé plus de paille en fin d'expérimentation, par rapport à la période initiale.

\section{Méthodes d'analyses}

Les digestibilités ont été déterminées après récolte totale des fèces pendant 5 jours.

Les prélèvements quotidiens de 10 p. 100 du total des matières fécales ont été aspergés avec une solution de $\mathrm{H}_{2} \mathrm{SO}_{4}$ à 2 p. 100 afin de limiter les pertes d'azote; ils ont été ensuite homogénéisés; une partie a été séchée à $80^{\circ} \mathrm{C}$ pendant 72 heures puis broyée et conservée en chambre froide jusqu'au moment des analyses. On a recueilli de même 5 p. 100 des urines acidifiées, en vue des mesures de bilans azotés. 


\section{Tableau 2}

Poids des animaux et quantités journalières ingérées (en brut).

Weight of animals and daily feed intake.

\begin{tabular}{|c|c|c|c|c|c|}
\hline \multirow{2}{*}{\multicolumn{2}{|c|}{ Régimes - Diets }} & \multicolumn{4}{|c|}{ Animaux - Animals } \\
\hline & & A & B & $\mathrm{C}$ & $\mathrm{D}$ \\
\hline \multirow{3}{*}{$\begin{array}{l}\text { I. Paille sodée } \\
\text { (P.S.) } \\
\text { ad libitum } \\
\mathrm{NaOH} \text { straw }\end{array}$} & PV (en kg) & 185 & 195 & 174 & 200 \\
\hline & Ingestion $(\mathrm{g} / \mathrm{Al} / \mathrm{j})$ & 2950 & 3410 & 1970 & 4000 \\
\hline & Ingestion $\left(\mathrm{g} / \mathrm{kg} \mathrm{PV}^{0,75} / \mathrm{j}\right) \ldots$ & 59 & 65 & 41 & 75 \\
\hline \multirow{4}{*}{$\begin{array}{l}\text { II. Paille sodée } \\
+ \text { tourteau de soja } \\
\text { (P.S. + T.S.) } \\
\text { ad libitum } \\
\text { NaOH straw } \\
+ \text { soybean meal }\end{array}$} & PV (en $\mathrm{kg}) \ldots \ldots \ldots$ & 185 & 192 & 164 & 184 \\
\hline & Ingestion $\mid$ totale $\ldots \ldots \ldots$ & 4100 & 5100 & 3000 & 5500 \\
\hline & $(\mathrm{g} / \mathrm{Al} / \mathrm{j}) \mid$ P.S. .... & 3530 & 4380 & 2580 & 4710 \\
\hline & $\begin{array}{l}\text { Ingestion (en P.S.) } \\
\left(\mathrm{g} / \mathrm{kg} \mathrm{PV} \mathrm{P}^{0,75} / \mathrm{j}\right) \ldots \ldots\end{array}$ & 70 & 85 & 56 & 95 \\
\hline \multirow{3}{*}{$\begin{array}{l}\text { III. P.S. + T.S. } \\
\text { q. limitée - restricted }\end{array}$} & PV (en $\mathrm{kg}) \ldots . .$. & 192 & 198 & 178 & 202 \\
\hline & \begin{tabular}{l|l} 
Ingestion & totale $\ldots \ldots \ldots \ldots$ \\
$(\mathrm{g} / \mathrm{Al} / \mathrm{j})$ & P.S. $\ldots \ldots \ldots \ldots$
\end{tabular} & $\begin{array}{l}2500 \\
2150\end{array}$ & $\begin{array}{l}25,00 \\
2150\end{array}$ & $\begin{array}{l}2500 \\
2150\end{array}$ & $\begin{array}{l}2500 \\
2150\end{array}$ \\
\hline & $\begin{array}{l}\text { Ingestion (en P.S.) } \\
\left(\mathrm{g} / \mathrm{kg} \mathrm{PV} \mathrm{PV}^{0,75} / \mathrm{j}\right) \ldots \ldots \ldots \ldots\end{array}$ & 42 & 41 & 45 & 40 \\
\hline \multirow{3}{*}{$\begin{array}{l}\text { IV. P.S. } \\
\text { q. limitée - restricted }\end{array}$} & PV (en $\mathrm{kg}$ ) ..... & 186 & 190 & 173 & 193 \\
\hline & Ingestion $(\mathrm{g} / \mathrm{Al} / \mathrm{j}) \ldots \ldots$ & 2200 & 2200 & 2200 & 2200 \\
\hline & Ingestion $\left(\mathrm{g} / \mathrm{kg} \mathrm{PV} V^{0,75} / \mathrm{j}\right)$ & 44 & 43 & 45 & 42 \\
\hline
\end{tabular}
vantes :

Les échantillons, aliments, fèces et urines, ont été soumis aux analyses sui-

- MAT $(\mathrm{N} \times 6,25)$, par la méthode de Kjeldahl ;

- cellulose brute, par la méthode de Weende;

- constituants pariétaux, selon la méthode de Van Soest (1963);

- matières minérales totales, après incinération à $550^{\circ} \mathrm{C}$ pendant 5 heures;

- calcium, par spectrophotométrie d'absorption atomique;

- sodium par spectrophotométrie d'émission de flamme;

— phosphore, par spectrophotométrie à $430 \mathrm{~nm}$ avec le réactif vanadomolybdique.

Les résultats expérimentaux sont soumis aux tests suivants selon Schwartz (1969) : le test « $t$ »de Student, la méthode des couples, l'analyse de variance simple avec test « $\mathrm{t}$ multiple protégé, et l'analyse de variance plurifactorielle avec ou sans interaction qui teste les effets spécifiques. 


\section{Résultats et discussion}

L'appétibilité de la paille traitée à la soude est d’abord satisfaisante, puis décline progressivement au cours de la première période. La complémentation azotée a permis d'accroître la quantité ingérée, en même temps que la digestibilité, et profite donc doublement à la valeur nutritive de la paille (ingéré $\times$ digéré).

Parallèlement, la consommation de paille traitée réhausse sensiblement l’ingestion d'eau et la production d'urine, sans que nous ayions pu mesurer le volume de chacune d'elles.

Pour les régimes (paille «sodée» + tourteau de soja $=$ PS + TS), et pour la paille «sodée » seule, les résultats relatifs aux digestibilités apparentes (moyennes, avec écarts-types) sont récapitulés dans le tableau 3.

Les coefficients d'utilisation digestive (CUD) adoptés pour le tourteau de soja, afin de calculer les CUD de la paille «sodée» des régimes composés, sont ceux déterminés par l'INRA (1978) pour les ruminants, à savoir 0,92 pour l'azote, 0,91 pour la matière organique et 0,80 pour la cellulose et les constituants pariétaux (excepté la lignine considérée comme totalement indigestible), soit environ 0,85 pour la matière sèche. Par comparaison, on a pu supposer que la digestibilité de cette dernière chez le cheval se situe approximativement vers 0,80 .

Le CUD de la paille «sodée» du régime a été obtenu au moyen de la relation suivante :

$$
\text { CUD régime }=\text { CUD P.S. } \times A+\text { CUD T.S. } \times \text { B }(1-\mathrm{A})
$$

avec $\mathrm{A}=$ pourcentage de l'élément considéré en provenance de la paille «sodée » dans la ration.

On peut remarquer la plus forte variabilité individuelle des résultats pour les périodes 3 et 4 , pendant lesquelles les animaux sont en alimentation restreinte.

Les digestibilités de la paille non traitée qui sert de référence (Wolter et al., 1979) se situaient au même niveau d'ingestion restreint que pour les périodes 3 et 4 .

\section{A. Digestibilité des régimes}

L'adjonction de tourteau de soja à la paille sodée améliore la digestibilité apparente de l'azote mais diminue celle de la lignine (tabl. 3 a - comparaison des régimes I + IV et II + III). Aussi, la lignine comme marqueur inerte de la digestibilité semble sujet à caution, comme l'avaient déjà signalé FaHEY, MCLarEN \& Williams (1979), car en fonction de la source végétale, elle peut s'avérer résistante ou labile aux mêmes traitements chimiques. De plus, selon la méthode de dosage utilisée, les teneurs en lignine peuvent être très variables.

La digestibilité des minéraux (Ca, $\mathrm{Na}, \mathrm{P} \ldots$...) est difficilement interprétable du fait que l'abreuvement n'était pas contrôlé et que l'analyse de l'eau a révélé des teneurs minérales inégales. Nous l'avons notée pour mémoire mais nous nous garderons d'en tirer des conclusions. 


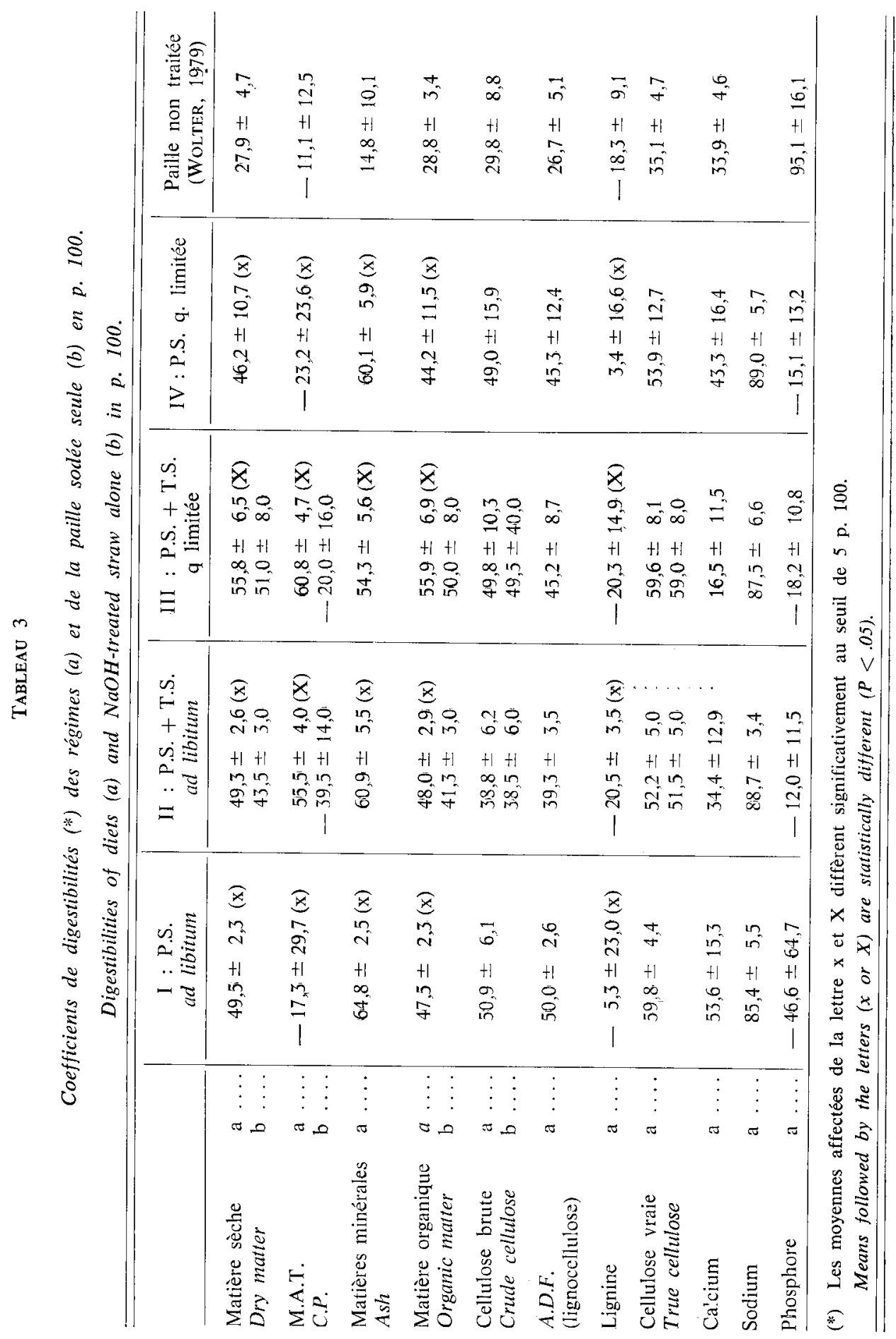


Le tableau 4 récapitule les effets du tourteau de soja et du niveau d'alimentation sur la digestibilité des régimes.

L'étude de ces effets montre que le niveau d'alimentation n'a aucune action par lui-même. Le tourteau de soja améliore bien entendu la digestibilité de l'azote, de la matière sèche et de la matière organique. Par contre, il diminue la digestibilité de la lignocellulose et bien sûr de la lignine. L'interaction signifie, dans notre étude, que la différence des moyennes observées entre les deux niveaux de l'effet «soja» (présence ou absence de tourteau de soja dans le régime) n'est pas la même selon les deux niveaux de l'effet «niveau d'alimentation» (limité ou ad libitum).

\section{TABleau 4}

Etude des effets sur la digestibilité des régimes.

Study of effects upon diets digestibilities.

\begin{tabular}{|c|c|c|c|}
\hline & $\begin{array}{c}\text { Effet « soja » } \\
\text { «Soybean » effect }\end{array}$ & $\begin{array}{c}\text { Effet « niveau } \\
\text { d'alimentation } \\
\text { Feeding level effect }\end{array}$ & Interaction \\
\hline Matière sèche - Dry matter & + & O & Oui - Yes \\
\hline Azote - Nitrogen & +++ & 0 & Non - No \\
\hline $\begin{array}{l}\text { Cellulose Weende } \ldots \ldots \ldots \ldots \\
\text { Crude cellulose }\end{array}$ & O & 0 & Oui - Yes \\
\hline Lignocellulose (A.D.F.) ..... & - & O & Non - No \\
\hline Lignine (A.D.L.) $\ldots \ldots \ldots \ldots$ & -- & 0 & Non - No \\
\hline $\begin{array}{l}\text { Cellulose «vraie » } \\
\text { (A.D.F.-A.D.L.) }\end{array}$ & $\mathrm{O}$ & $\mathrm{O}$ & Oui - Yes \\
\hline $\begin{array}{l}\text { Matière organique } \ldots \\
\text { Organic matter }\end{array}$ & + & O & Oui - Yes \\
\hline
\end{tabular}

L'examen de ces interactions, très délicates, nous permet d'avancer que le tourteau de soja diminue la digestibilité de la cellulose essentiellement en alimentation ad libitum, mais que, associé à un bas niveau de consommation, il améliore nettement la digestibilité de la matière sèche et de la matière organique.

\section{B. Digestibilité de la paille «sodée» des régimes}

Le tableau $3 \mathrm{~b}$ rapporte les CUD de la paille sodée seule, calculée d'après la formule précédemment indiquée.

En tout état de cause, les tables de digestibilité et de valeur alimentaire établies chez les ruminants conviennent mal aux équidés, d'autant plus que l'aliment est 
plus cellulosique, à cause des différences anatomophysiologiques, qui existent entre les deux espèces; nous aurions dû établir les CUD du tourteau de soja chez les équidés en utilisant plusieurs taux d'incorporation dans les rations et en traçant la droite de régression :

$$
\text { CUD régime }=\text { pente } \mathrm{X}(1-\mathrm{A})+\text { CUD P.S. }
$$

Nous aurions obtenu pour $1-\mathrm{A}=0$ la digestibilité de la paille «sodée » seule et pour $1-\mathrm{A}=1(\mathrm{~A}=0)$ la digestibilité du tourfeau de soja.

De même, nous avons négligé le phénomène de digestibilité associative qui a pu se produire lors de la substitution.

Contrairement aux constatations faites avec les régimes, on n'observe pas alors d'effets directs dus au soja mais on note des interactions pour la matière sèche, la matière organique et la cellulose vraie. L'adjonction de tourteau de soja améliore la digestibilité de la paille, seulement quand celle-ci est distribuée de façon restreinte.

La comparaison avec les digestibilités de la paille de blé non traitée (même si les conditions de climat et de récolte modifient la valeur de différents lots de pailles et limitent la signification d'une telle comparaison) (Wolter et al., 1979) semble montrer l'amélioration due au traitement à la soude. La digestibilité de la matière sèche passe de 28 à 48 p. 100, et celle de la matière organique de 29 à 48 p. 100, pour la paille seule. Slagswold, Hintz \& Schryver, 1979, ont également mis en évidence que le traitement à l'ammoniaque élève la digestibilité de la matière sèche de la paille de 35 p. 100 à 48 p. 100.

Pour JACKSON (1977), le traitement aux alcalis désorganise les parois cellulaires en dissolvant la lignine et la silice et en hydrolysant les esters des acides uroniques et acétiques des hémicelluloses, ce qui entraîne l'amélioration de la digestibilité de la cellulose. Dans nos essais, celle-ci progresse de 30-35 p. 100 à 50-60 p. 100. A ce propos, on peut remarquer que la cellulose «vraie» est plus digestible que la cellulose Weende (de 5 à 10 points).

La digestibilité de l'azote est toujours mauvaise, que la paille soit traitée ou non, mais l'adjonction d'azote diminue la digestibilité de la cellulose de la paille traitée alors que l'on n'observe pas de différence avec la paille non traitée (SLAGSwold, HiNTZ \& SCHRYVER, 1979).

\section{Bilans azotés (tabl. 5)}

Pour les quatre régimes, les bilans sont respectivement de $-116,232,263$ et $-153 \mathrm{mg} / \mathrm{kg} \mathrm{PV}^{0,75}$, ce qui s'explique par le fait que les régimes I et IV ne contiennent pas de complément d'azote.

L'équation de régression de la rétention (y) en fonction de l'azote absorbé (x) est :

$$
\mathrm{y}=-174+0,600 \mathrm{x} \quad(\mathrm{r} / 14 \mathrm{ddl}=0,95 \mathrm{~s} \text { à } 1 \text { p. } 1000) .
$$

Il faut $290 \mathrm{mg} \mathrm{N} / \mathrm{kg} \mathrm{PV}^{0,75}$ pour équilibrer le bilan azoté $(\mathrm{y}=0$ ). L'azote urinaire endogène est estimé en posant $\mathrm{x}=0$, et la valeur obtenue, $174 \mathrm{mg} / \mathrm{kg} \mathrm{PV} \mathbf{0 , 7 5}$ est en bon accord avec celles qui sont généralement admises (SLade, Robinson \& CaSEY, 1970 ; Reitnour \& Treece, 1971 ; Hintz \& Schryver, 1972). 
L'efficacité d'utilisation (E.U.) de l'azote absorbé (*) peut done être calculée à partir de la formule :

$$
\begin{gathered}
\text { E.U.N. }=100 \times \frac{N \text { absorbé }-(N \text { urinaire }-N \text { urinaire endogène })}{N \text { absorbé }} \\
\quad \text { ou }: 100 \times \frac{N \text { retenu }+N \text { urinaire endogène }}{N \text { absorbé }}
\end{gathered}
$$

TABLEAU 5

Bilans azotés (moyennes des quatre animaux).

\begin{tabular}{|c|c|c|c|c|c|c|c|}
\hline \multirow[b]{2}{*}{$\begin{array}{c}\text { Régime } \\
\text { Diet }\end{array}$} & & \multicolumn{5}{|c|}{$\operatorname{mg~N} / \mathrm{kg} P V^{\mathrm{s}, \pi \mathrm{s}}$} & \multirow{2}{*}{$\begin{array}{c}\text { «Efficacité } \\
\text { azotée » (*) } \\
\text { Nitrogen } \\
\text { efficiency }\end{array}$} \\
\hline & & $\begin{array}{l}\mathrm{N} \text { ingéré } \\
\text { Inges- } \\
\text { ted } N\end{array}$ & $\begin{array}{c}N \text { fécal } \\
\text { Faecal } N\end{array}$ & $\begin{array}{l}N \text { uri- } \\
\text { naire } \\
\text { Urinary } \\
N\end{array}$ & $\begin{array}{c}\mathrm{N} \\
\text { absorbé } \\
\mathrm{I}-\mathrm{F} \\
\text { Absorbed } \\
N\end{array}$ & $\begin{array}{c}\mathrm{N} \text { retenu } \\
\mathrm{I}- \\
(\mathrm{F}+\mathrm{U}) \\
\text { Retained } \\
N\end{array}$ & \\
\hline \multicolumn{2}{|l|}{ P.S. (ad libitum) } & 272 & 319 & 69 & -47 & -116 & -123 \\
\hline \multicolumn{2}{|l|}{ P.S. (q. limitée) } & 177 & 218 & 112 & -41 & -153 & -51 \\
\hline \multicolumn{2}{|l|}{$\begin{array}{l}\text { P.S. + T.S. } \\
(\text { ad libitum })\end{array}$} & 1330 & 590 & 508 & 740 & 232 & 54,9 \\
\hline \multicolumn{2}{|l|}{$\begin{array}{l}\text { P.S. + T.S. } \\
\text { (q. limitée) }\end{array}$} & 1.043 & 368 & 412 & 675 & 263 & 64,7 \\
\hline \multirow{2}{*}{ (*) $\mathbf{E U N}=100 \times$} & \multicolumn{5}{|c|}{$N$ absorbé $-(N$ urinaire $-N$ urinaire endogène $)$} & & \\
\hline & \multicolumn{5}{|c|}{$\mathbf{N}$ absorbé } & & \\
\hline \multirow{2}{*}{$E U N=100 \times$} & \multicolumn{5}{|c|}{ Absorbed $N$-(urinary $N$-endogenous urinary $N)$} & & \\
\hline & \multicolumn{5}{|c|}{ Absorbed $N$} & & \\
\hline
\end{tabular}

Nitrogen balances (means of four animals).

Les valeurs obtenues montrent que l'azote est mieux utilisé en alimentation restreinte qu'en alimentation ad libitum. Pour les régimes à base de paille «sodée » seule, l'interprétation est plus délicate du fait que le bilan azoté est fortement négatif.

(*) Notion différente de la valeur biologique car ne tenant pas compte de l'azote fécal endogène que nous n'avons pas pu déterminer expérimentalement. 


\section{Conclusion}

La paille broyée, agglomérée, traitée à la soude puis granulée est plus appétente, mieux consommée et plus digestible, que le produit de base.

La paille «sodée» seule ne permet pas de couvrir les besoins d'entretien chez le poney, mais, après complémentation en azote, sa valeur nutritive est comparable à celle d'un foin de qualité moyenne.

Son utilisation, associée à un aliment concentré ou à un ensilage d'herbe, peut être envisagée dans l'alimentation des équidés en prenant garde toutefois aux problèmes que pourrait poser l'excès de sodium.

Bien entendu, des essais zootechniques, portant sur des chevaux et s'adressant à un plus grand nombre d'animaux, restent nécessaires pour mieux préciser l'appétibilité, l'innocuité, la digestibilité et la valeur énergétique d'une telle paille traitée aux alcalis.

Accepté pour publication en octobre 1982.

\section{Summary \\ Digestibility of $\mathrm{NaOH}$-treated straw in ponies}

The NaOH-treated, pelleted straw was given to four ponies in ad libitum (40 to $94 \mathrm{~g} / \mathrm{kg} \mathrm{PV}^{0.75}$ ) or restricted (near $42 \mathrm{~g} / \mathrm{kg} \mathrm{PV}^{0.75}$ ) amounts. The ponies, placed in metabolism crates, were fed with the straw alone or with soyabean meal (Tables 1 and 2).

Apparent digestibilities of the diets (Table 3) showed the positive effect of the treatment of the straw as compared with non treated straw. Food restriction alone had no effect, but the soyabean supplementation (Table 4) had a benefic effect upon diet digestibility, associated with a low level of feeding, but did not affect straw digestibility itself.

The nitrogen balance (Table 5) showed an improvement of nitrogen utilization in restricted feeding experiments.

\section{Références bibliographiques}

Capper B.S., Morgan D.S., Parr W.H., 1977. Alkali-treated roughages for feeding ruminants : a review. Trop. Sci., 19, 73-87.

Dulphy J.P., Gomez Cabrera A., 1977. Utilisation des pailles traitées à la soude par les bovins. Bull. tech. C.R.Z.V.Theix, I.N.R.A., 30, 23-34.

DulPhy J.P., ANDrieu J.P., 1980. Valeur alimentaire de pailles traitées à la soude pour des génisses de 2 ans. Bull. tech. C.R.Z.V. Theix, I.N.R.A., 39, 11-14.

Fahey G.C., McLaren G.A., Williams J.E., 1979. Lignin digestibility by lambs fed both low quality and high quality roughages. J. anim. Sci., 48, 941-946.

FEIST W.C., Baker A.S., Tarkow H., 1970. Alkali requirements for improving digestibility of hardwoods by rumen micro-organisms. J. anim. Sci., 30, 832-835.

Hintz H.F., Schryver H.F., 1972. Nitrogen utilization in ponies. J. anim. Sci., 34, 592595. 
I.N.R.A., 1978. Alimentation des ruminants. Ed. I.N.R.A. Publications (route de SaintCyr), 78000 Versailles, 552-553.

JACKson M.G., 1977. Review Article : the alkali treatment of straws, Anim. Feed Sci. Technol., 2, 105-130.

JACKson M.G., 1978. Traitement des pailles pour l'alimentation animale. Evaluation technique et économique. Rev. mond. Zootech., 28, 38-43.

Johnson R.J., HART J.W., 1974. Utilization of nitrogen from soybean meal, biuret and urea by equine. Nutr. Rep. Intern., 9, 209-216.

JoUANy J.P., 1975. Etudes des traitements permettant d'améliorer la valeur alimentaire des fourrages pauvres (pailles). Bull. tech. C.R.Z.V. Theix, I.N.R.A., 21, 5-15.

Meyer H., Mundt H.C., 1980. Treated straw in horse nutrition. Prakt. Tierärztl., 61, 206-214.

Meyer H., Schmidt M., Güldennhaupt V., 1981. Untersuchung über Mischfutter für Pferde. Dischl. Tierärztl. Wschr., 88, 2-5.

Mundt H.C., 1978. Untersuchung über die Verdanlichkeit von aufgeschlossenem Stroh beim Pferd. Thesis-Tierärztliche Hochschule Hannover, Deutschland, 109 p.

Reitnour C.M., Treece J.M., 1971. Relationship of nitrogen source to certain blood components and nitrogen balance in the equine. J. anim. Sci., 32, 487-492.

Schwartz D., 1969. Méthodes statistiques à l'usage des médecins et des biologistes. Flammarion Médecine. Sciences, Paris, 318 p.

Slade L.M., Robinson D.W., CASEY K.E., 1970. Nitrogen metabolism in non ruminant herbivores. I. - The influence of nonprotein nitrogen and protein quality on the nitrogen retention of adult mares. J. anim. Sci., 30, 753-760.

Slagswold P., Hintz H.F., Schryver H.F., 1979. Digestibility by ponies of oat straw treated with anhydrous ammonia. Anim. Prod., 28, 347-352.

Van Hoecke P.P., Cottyn B.G., 1979. Comment améliorer la valeur alimentaire de la paille, utilisée comme aliment pour le bétail. Etude bibliographique. I. - Traitement à la soude. Rev. Agric., 32, 71-93.

VAN Soest P.J., 1963. Use of detergent in the analysis of fibrous fed. II. - A rapid method for the determination of fiber and lignin. J. Assoc. off. anal. Chem., 46, 829-835.

Volgt J., Piatkowski B., 1974. Untersuchungen zum Aufschluss von Getreidestroh. Arch. Tierernähr., 24, 589-600.

Wolter R., Durix A., Letourneau J.C., 1975. Influence du mode de présentation du fourrage sur la digestibilité chez le poney. Ann. Zootech., 24, 237-242.

Wolter R., Durix A., Letourneau J.C., Carcelen M., 1979. Evaluation chez le poney de la digestibilité du maïs-fourrage déshydraté, des pulpes sèches de betterave, de la luzerne déshydratée, du son de blé, de la paille de blé et des pulpes de raisins. Ann. Zootech., 28, 93-100.

Wolter R., Durix A., Letourneau J.C., Carcelen M., 1980. Evaluation chez le poney de la digestibilité des pellicules de soja, du marc de pommes, des caroubes et du tourteau de pépins de raisins. Ann. Zootech., 29, 377-385. 